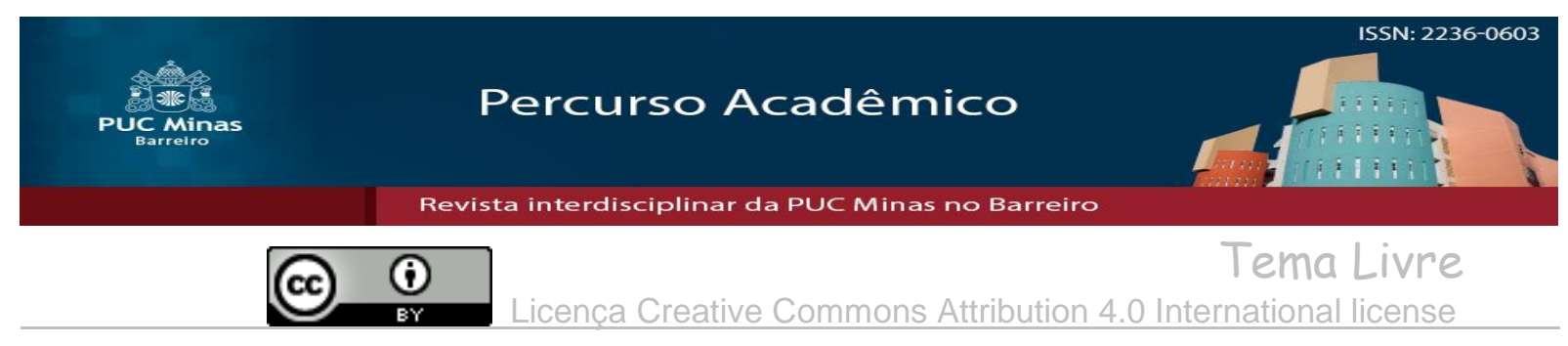

\title{
Atuação das equipes de atenção domiciliar nos cuidados paliativos
}

\author{
The role of the home care team in palliative care
}

\author{
Aislaine Junia Oliveira ${ }^{1}$ \\ Andrea Lemos Ribeiro ${ }^{2}$ \\ José Sérgio Domingos de Lima ${ }^{3}$ \\ Natália de Cássia Horta ${ }^{4}$
}

\section{Resumo:}

No âmbito da atenção domiciliar os cuidados paliativos são uma abordagem terapêutica que tem como objetivo promover a melhoria da qualidade de vida a pacientes e familiares diante de uma doença que ameace a vida, proporcionando alívio da dor e de outros sintomas. Objetivo: Descrever a atuação das equipes de atenção domiciliar em cuidados paliativos, bem como conhecer os desafios e limites dos profissionais nessa modalidade de cuidado Metodologia: Trata de pesquisa descritiva, de abordagem qualitativa, desenvolvida em um município de grande porte da Região Metropolitana de Belo Horizonte, por meio de questionário. Participaram 12 profissionais do programa de Atenção domiciliar deste município, sendo feita análise de conteúdo. Resultados: A análise permitiu a construção das seguintes categorias: "Cuidados paliativos: a concepção e a pratica; Construção do plano de cuidado e a relação com a família e cuidador" trazendo a realidade da assistência prestada e "Rede de saúde e cuidados: desafios e recomendações" mostrando as possibilidades e fragilidades na prestação da assistência a saúde. Considerações finais: Os profissionais reconhecem a importância dos cuidados paliativos na atenção domiciliar. Entretanto atuam de forma limitada devido à necessidade de capacitação em cuidados paliativos, de carência de recursos profissionais e insumos, corroborado pela precariedade socioeconômica da população.

Palavras-chave: Cuidados Paliativos; Serviços de Assistência; Equipe de Assistência ao Paciente.

\section{Abstract:}

Artigo recebido em 01 de dezembro de 2018 e aprovado em 04 de outubro de 2019.

${ }^{1}$ Graduação em Enfermagem pela Pontifícia Universidade Católica de Minas Gerais, Betim, MG Brasil (2018). E-mail: aislaineoliveira@yahoo.com.br

${ }^{2}$ Graduação em Enfermagem pela Pontifícia Universidade Católica de Minas Gerais, Betim, MG, Brasil (2018). E-mail: andrealemos@ @otmail.com

${ }^{3}$ Graduando do curso de Enfermagem Pontifícia Universidade Católica de Minas Gerais, Betim, MG, Brasil. E-mail: j.sergio.d.lima@gmail.com

${ }^{4}$ Doutora em enfermagem. Docente adjunto IV do Instituto de Ciências Biológicas e da Saúde e líder do grupo de pesquisa PHASE da Pontifícia Universidade Católica Minas Gerais, Brasil. E-

mail: nataliahorta@pucminas.br 


\begin{abstract}
In the scope of the home care services, the palliative care is considered a therapeutic approach that aims to promote the quality of life of terminal illness' patients and their families, in the face of diseases that threaten their lives by providing relief from pain and other symptoms. Objective: To describe the role of the home care teams in palliative care; to understand the challenges and limitations of healthcare professionals in palliative care. Methodology: This is a descriptive qualitative study conducted in a large city in the Metropolitan Area of Belo Horizonte. A structured questionnaire was used for data collection. The participants were 12 healthcare personnel that belong to the home care team of the mentioned city and content analysis was performed. Results: From the data analysis the following categories emerged: "Palliative care: the theory and practice"; "The plan of care and the collaboration with family and caregiver", which illustrates the healthcare delivered, and "Healthcare and network services :: challenges and recommendations" highlighting healthcare opportunities and drawbacks.in healthcare delivering. .Final considerations: The healthcare professionals recognize the importance of palliative care in home care services. However, their role is limited due to lack of training, short staff, and lack of supplies and materials, in addition to the problems related to the low socioeconomic status of the population.
\end{abstract}

Keywords: Palliative care; Home Care Services; Patient care team.

\title{
Introdução
}

Entende-se como cuidados paliativos na assistência domiciliar, aqueles prestados aos pacientes e familiares que se encontram fragilizados, incluindo aqueles que vivenciam a terminalidade da vida. Cuidados estes, mediados pela equipe multiprofissional, objetivando orientar os cuidadores, a fim de proporcionar ao paciente maior qualidade em sua respectiva condição. A Organização Mundial de Saúde (OMS), em 1990, definia cuidados paliativos como assistência terapêutica promovida por uma equipe multidisciplinar que atua de forma humanizada com paciente com doença já em fase avançada sem perspectiva de cura. Este conceito foi redefinido em 2002, sendo esta abordagem terapêutica estendida do paciente aos familiares, objetivando a melhoria da qualidade de vida e do sofrimento diante de uma doença que ameace a vida. Por meio de prevenção, requer a identificação precoce, avaliação e tratamento da dor e outros problemas psicossociais e espirituais. (ANCP, 2012; WHO,2018).

As ações dos cuidados paliativos redefinidas pela OMS em 2002 apontam também que se deve respeitar a ideia de que a finitude é um ciclo natural da vida, não prolongar ou antecipar a morte, garantir ao paciente um suporte que o permita viver mais ativamente possível, oferece praticas integrativas, psicossocial e espiritual, além da atuação conjunta a outras medidas de tratamento. (BRASIL, 2013). 
No âmbito do SUS a atenção domiciliar é dividida em três modalidades: AD1, AD2 e AD3 que são referenciadas de acordo com o grau de peculiaridades dos pacientes. Na AD1 geralmente os pacientes são de baixa complexidade e demandam visitas menos frequentes e estas são realizadas pela Estratégia de Saúde da Família (ESF). As modalidades AD2 e AD3 são específicas do Serviço de Atenção Domiciliar (SAD), intitulado pela instância federal como Melhor em Casa, pois demandam cuidados com grau de complexidade maior e visitas mais frequentes. O SAD é organizado em equipes que são identificadas em Equipe Multiprofissional de Atenção Domiciliar (EMAD) e Equipe Multiprofissional de Apoio (EMAP). A primeira é composta por médicos, enfermeiros, técnicos ou auxiliar de enfermagem, fisioterapeutas ou assistente social e a segunda, por no mínimo três profissionais de nível superior, técnico ou auxiliar de enfermagem. (BRASIL, 2015)

No que se refere aos cuidados paliativos neste contexto, a função de todos os profissionais da equipe é de prestar assistência de qualidade e dar apoio, suporte, carinho, dizer a verdade sem tirar a esperança, e ajudar o paciente em seu luto, medo, preocupações, raiva e frustações, o que requer conhecimento acerca dos cuidados paliativos. (BRASIL, 2015; OLIVEIRA, 2016). As principais práticas intervencionistas aplicadas a estes pacientes estão relacionadas às orientações, aconselhamento ao doente, cuidador e familiares, sobre o plano de cuidados, prepará-los para o enfrentamento da doença e sobre a terminalidade, controle da dor a partir de técnicas e métodos alternativos; controle de dispneia e sensação de asfixia com oxigenoterapia, aspirações, náuseas e vômitos através de uma alimentação tolerável ao paciente e uso de antieméticos, anorexia com avaliação nutricional, incentivo à hidratação, uso de antidepressivos, carinho e afeto entre outros. (OLIVEIRA, 2016)

Para promover uma assistência integral deve-se observar os principais pontos as serem trabalhados na formação dos profissionais, para que as equipes dos programas de atenção à saúde consigam levar um atendimento integral e humanizado aos pacientes, aos cuidadores e familiares. É necessário que dentro das abordagens no cuidado paliativo, reafirme-se como estes profissionais deverão servir de apoio e serem comprometidos com toda equipe, não ignorando os aspectos espirituais que aumentam a confiança trazendo segurança. Considera-se que o cuidado espiritual é uma forma de minimizar o sofrimento dos pacientes e familiares, para uma interação efetiva entre 
todos os envolvidos. Espera-se ainda que seja aprimoradoo grau de importância da boa comunicação e empatia por parte das equipes, para com os pacientes/familiares, de forma a adquirirem confiança, compreensão nas orientações e nos cuidados prestados por parte dos profissionais para que possam desenvolver um trabalho com resultados e benefícios cada vez maiores para o paciente. Assim, espera-se que a equipe consiga abordar e mostrar os aspectos positivos e os benefícios do cuidado paliativo na atenção domiciliar. (HERMES; LAMARCA, 2013; ARRIEIRA et al, 2017).

Neste sentido, este estudo foi norteado pelo seguinte questionamento: como a equipe da atenção domiciliar atua na prestação dos cuidados paliativos? Partiu-se do pressuposto de que a equipe atua de modo limitado, uma vez que os profissionais de saúde não estão preparados para prestarem os cuidados paliativos, sendo estes ainda incipientes, seja pela formação técnica, seja pelos insumos necessários para a prestação do cuidado. Teve como objetivo geral descrever a atuação da equipe de atenção domiciliar em cuidados paliativos, bem como os desafios vividos para esta assistência. Especificamente buscou-se: caracterizar o perfil dos profissionais da equipe na atenção domiciliar; identificar os desafios dos profissionais de saúde em lidar com as demandas dos pacientes em cuidados paliativos e familiares; identificar as falhas do serviço de saúde que refletem na descontinuidade da assistência pelos profissionais da equipe e da rede de atenção.

Observando a transição demográfica que vem ocorrendo no cenário brasileiro, devido ao aumento da expectativa de vida da população há um crescimento de doenças crônicas incuráveis, doenças neurológicas progressivas entre outras que vem demandando dos serviços de saúde a ampliação de cuidados paliativos, inclusive na atenção domiciliar. (HERMES; LAMARCA,2013). Além disso, o modelo de assistência domiciliar impacta na alta hospitalar de pacientes portadores de doenças não curáveis e vem exigindo que os profissionais de saúde estejam mais preparados, pois existem desafios a serem superados na atuação destes. Entretanto, evidencia-se o despreparo das equipes multiprofissionais na atuação das práticas, processos de humanização, aceitação da finitude humana, falta de manejo pessoal quanto ao processo de terminalidade. (PILATI et al, 2017). 
Este estudo é importante uma vez que reafirma que os cuidados paliativos na assistência domiciliar contribuem para o aumento da qualidade do cuidado, garantindo conforto e autonomia do paciente e seus familiares, diminuindo a hospitalização, consequentemente o risco de infecção hospitalar e gastos financeiros. (VASCONCELOS; PEREIRA, 2018).

É importante ressaltar que a ação de uma equipe multidisciplinar em que cada profissional reconhece seu limite, se desprenda do tradicional conceito de saúde e compreenda que o objetivo desse cuidado é valorizar os diferentes reconhecimentos envolvidos que poderá contribuir para melhor assistência ao controle da dor e dos sintomas, sendo estes o foco para o cuidado individual, além dos aspectos físico, mental, espiritual e social. (CREMESP, 2008)

\section{Metodologia}

Trata-se de pesquisa descritiva, de caráter qualitativo que, segundo Minayo (2007) tem o objetivo de compreender os fenômenos humanos envolvendo suas ações baseadas em seus valores, sentimentos e experiências, inseridas em sua realidade onde os aspectos culturais, econômicos sociais e históricos não são passíveis de controle, permitindo uma interpretação a partir dos próprios sujeitos envolvidos na situação.

A pesquisa aconteceu em um município de grande porte da Região Metropolitana de Minas Gerais. Neste contexto, a rede de Atenção à Saúde (RAS) conta com a Atenção Primária à Saúde (APS) organizada dentro das diretrizes da Estratégia de Saúde da Família (EAS), como ordenadora da assistência, viabilizando a integralidade da atenção, a qualificação das práticas e a gestão do cuidado. É composta por diferentes serviços que incluem: 4 unidades de urgência e emergência, 35 unidades de atenção primária com 120 equipes, sendo 78 equipes de saúde da família, 2 hospitais, 1 maternidade pública, 5 centros de referência em atenção especializada e o Serviço de Atenção Domiciliar.

Atenção Domiciliar foi implantada no município desde a década de 1990, com a denominação Programa de Internação Domiciliar (PID). Passou a ser oferecida como Serviço de Atenção Domiciliar no ano de 2009, a partir da Unidade de Atenção Imediata (UAI), porta de entrada para os casos de urgência e emergência, pelo PID e pelo Programa de Atendimento Domiciliar (PAD). Com a criação do programa federal, 
intitulado "Melhor em Casa", o município reorganizou o processo de trabalho das equipes de assistência domiciliar e conta com 4 EMADs em Unidade de Urgência e Emergência (UAI Sete de Setembro, UAI Guanabara, UAI Teresópolis e UAI Alterosa) e 1 EMAP nestas equipes atuam 21 profissionais do nível médio e 25 de nível superior com as seguintes formações: enfermeiros, médicos generalistas, médico pediatra, assistentes sociais, fonoaudiólogos, fisioterapeutas, nutricionistas e cirurgião dentista.

A coleta de dados ocorreu entre os dias 29 de outubro a 14 e novembro de 2018 por meio de questionário, contendo dezoito questões abertas e fechadas, sobre a prática profissional na atenção domiciliar que entregues em todas as equipes, obedecendo ao quantitativo de profissionais por equipe. Após a entrega dos questionários foram realizados contatos pessoalmente e telefônicos diários com as equipes a fim de orientálos sobre a pesquisa e motivar a participação.

A pesquisa foi submetida à aprovação do Comitê de Ética em Pesquisa com Seres Humanos da Pontifícia Universidade Católica de Minas Gerais e da Prefeitura Municipal de Betim, sob n CAAE 096859318.9.00005137. Participaram da pesquisa, doze profissionais após a leitura e assinatura do Termo de Consentimento Livre e Esclarecido, conforme a Resolução 466/12, do Conselho Nacional de Saúde. O critério de participação na pesquisa foi de ter, no mínimo, seis meses de atuação na atenção domiciliar. Deste modo, dentre os 46 profissionais distribuídos em cinco equipes no município, a pesquisa contou com 12 participantes de três das equipes, sendo que dois profissionais não puderam participar por ter menos de seis meses de atuação na $\mathrm{AD}$, e dos demais, considerando o prazo estabelecido para o trabalho de campo, não foi possível obter o retorno.

A partir das respostas obtidas, foi realizada análise de conteúdo, conforme proposto por Minayo (2007). Esta técnica permite a confirmação ou não das afirmativas estabelecidas pela hipótese, e também de descobertas que vão além do conteúdo e da aparência manifestada. Ela perpassa por três fases: a pré-análise (leitura compreensiva do material/organização do conteúdo), exploração do material (análise propriamente dita) e tratamento dos resultados (síntese interpretativa que permite dialogar temas com objetivos, questões e pressupostos da pesquisa). Inicialmente foi feita a codificação dos 
questionários e o compilado das respostas por questões, com a construção de três categorias empíricas.

\section{Resultados e discussão}

Os participantes da pesquisa foram 12 profissionais de saúde que compõem 3 Equipe Multiprofissional de Atenção Domiciliar EMADs do município, sendo eles: dois médicos, três enfermeiros, cinco auxiliares/técnicos de enfermagem e dois assistentes sociais, com tempo médio de atuação no SAD de 4 anos e 6 meses. O vínculo profissional dos participantes foi de concurso público, sendo apenas uma participante com vínculo empregatício por contrato, regido de Consolidação das Leis do Trabalho (CLT).

Dos 12 profissionais, oito eram de nível superior e sete tinham entre um a quatro dos seguintes cursos de pós-graduação: Enfermagem em ginecologia obstétrica, enfermagem cardiológica, saúde da família, educação em saúde, gestão social e saúde pública, residência em patologia clínica, medicina do trabalho, medicina antroposófica e mestrado em medicina natural. Em relação aos conhecimentos e qualificação profissional específicos em cuidados paliativos, 06 profissionais disseram não ter realizado e 06 profissionais afirmaram ter os seguintes cursos: Atenção domiciliar, Cuidados paliativos pelo UNA-SUS e participação em simpósios sobre o tema. A média de carga horária dos cursos realizados foi de 33 horas, variando de 08 horas até 80 horas.

A análise permitiu a construção de três categorias: Cuidados paliativos: a concepção e a prática da equipe; A construção do plano de cuidado e a relação com a família e cuidador; Rede de cuidados: desafios e recomendações.

\section{Cuidados paliativos: a concepção e a prática da equipe}

Os participantes entendem como cuidados paliativos a assistência prestada a pacientes que estejam diante de uma doença grave já em estado avançado, visando proporcionar qualidade de vida a estes e seus familiares, com foco no conforto físico, alívio da dor e do sofrimento e atendimento psicológico. Na concepção de cuidados 
paliativos, apenas um participante incluiu o apoio espiritual no cuidado, dimensão esta fundamental para a integralidade das ações cuidadoras. Esta prática foi incorporada visto que a crença exerce uma grande influência para os pacientes em lidar com o sofrimento, sendo um contribuinte diferencial na melhoria de sintomas em qualquer estágio da doença, proporcionando bem estar não só no momento da morte. (ARRIEIRA et al, 2018)

Destaca-se que a compaixão e empatia também foram apontadas como importantes no cuidado paliativo, conforme explicitado pela participante M1:

"Proteger, é uma forma de cuidado com o objetivo de oferecer conforto físico, psicológico e espiritual ao paciente e aos familiares.” (M 1)

Deste modo, percebe-se que a concepção de cuidado paliativo apontada pelos participantes do estudo relaciona-se à não possibilidade de cura, à gravidade da doença e terminalidade, mas para, além disso, às necessidades biopsicossociais dos pacientes, visão holística que gera melhoras na qualidade de vida e o percurso da doença. (ANCP, 2012)

Nestas equipes são acompanhados pacientes oncológicos já em fase avançada, portadores de demência, de acidente vascular encefálico e de doenças crônicas. Os sintomas clínicos apontados pelos participantes são bem variáveis: apresentam dor, dispneia, hipotensão, hiperglicemia, diarreia ou constipação intestinal, febre, prostração, lesão de pele e depressão. Entretanto, a dor ainda não recebe atenção devida na assistência em nosso país. É necessário que sejam criados programas de educação em relação a esta problemática e que o tema seja discutido, e inserido nos currículos de formação dos profissionais. (PESSINI, 2011) Deve atentar-se também aos sintomas de depressão, comuns devido à vulnerabilidade destes pacientes, não deixando-os sem tratamento adequados, o que reforça a necessidade da integralidade do cuidado e da atenção de profissionais especializados. (ANTONECHEN, DORO; 2016)

Dados do estudo apontam que para a construção do plano de cuidados, as necessidades dos pacientes são levantadas durante a visita domiciliar, pois partem da avaliação do estado geral do paciente envolvendo aspectos físicos, psicológico, emocional, incluindo o diagnóstico de base. A partir de então são definidas as ações de 
cuidados paliativos a serem prestados ao paciente e família, que são executadas de acordo com cada categoria profissional. Os profissionais de enfermagem executam ações de orientação aos familiares quanto à realização de curativos, mudança de decúbito, manejo de SNE, SVD, dieta, além de oferecer apoio e carinho. O discurso de uma das participantes da equipe de enfermagem destaca a não realização de procedimentos desnecessários, prática fundamental nos cuidados paliativos:

"Controle da dor, curativos em úlceras visando o conforto, apoio familiar, observação das condições de conforto, ausência de procedimentos invasivos desnecessários.” (E 3)

O assistente social fica responsável pela captação de recursos como equipamentos, transporte, restabelecimento de laços familiares, acesso aos medicamentos entre outros, conforme explicitado abaixo:

"Com a assistente social os recursos que garantam o estabelecimento do plano de cuidados: acesso a benefícios; equipamentos (cama hospitalar, aspirador, dieta especial) reunião com familiares; restabelecimento de laços; transporte para casos de intercorrências; acesso a medicamentos e aciono a rede assistencial quando necessário". (AS 2)

Diante das falas supracitadas podemos perceber o envolvimento destes profissionais em executar suas ações de forma humanizada aos pacientes e familiares pois relatam estarem atentos às demandas emocionais, sociais, demonstraram estarem abertos à escuta, a oferecem carinho e apoio, o que é apontado também na literatura como postura dos profissionais da atenção domiciliar. (OLIVEIRA, 2016)

A equipe multidisciplinar tem um papel importante na fundamentação dos cuidados paliativos, pois além de executar ações de assistência à saúde, ela deve atuar no domicilio objetivando aliviar o sofrimento físico, emocional, social e espiritual do paciente e de seus familiares (ANCP, 2012). Ainda na atuação da equipe, o médico é responsável pela clínica como prescrições de sedação, analgesia por hipodermóclise e antibioticoterapia.

“Controle da dor, sedação e hipodermóclise.” ME 2: 
Observa-se ainda a presença do modelo biomédico, formação voltada para o tratamento e o diagnóstico da doença, com a necessidade de incremento da discussão sobre cuidado paliativo na formação considerando que, as ações devem ter o foco principal no doente e não na doença, uma vez que as demandas estão para além do físico tendo que trabalhar o lado psicológico, social e espiritual. (HERMES, LAMARCA; 2013)

Abaixo um depoimento que explicita a construção do plano de cuidados individualizados, e ampliado à família, a depender das necessidades levantadas pelos profissionais:

"Cada paciente é único. Estudo um pouco o paciente no contexto familiar: muitas vezes ele tem um desejo de ver um familiar. A família precisa de uma atenção psicológica." (AS 1)

Quando se trata de qualidade de vida, a abordagem deve ser centrada no paciente e seus familiares, já que cada ser é único. Assim a equipe precisa ter um olhar humanizado e holístico para entender as necessidades biopsicossocial e espiritual que demanda o cuidado. (VASCONSELOS, PEREIRA; 2018)

\section{A construção do plano de cuidado e a relação com a família e cuidador}

O processo de construção do plano de cuidados foi apontado pelos participantes como uma ação da equipe, mais centrado nos profissionais de nível superior, e elaborado de modo colegiado:

"Por meio de discussões dentro da equipe, afim de que cada área (medicina, enfermagem, assistência social, fonoaudiologia, fisioterapia e nutrição) possa enriquecer e contribuir para o cuidado com ênfase no conforto.” (M 1)

Os participantes afirmaram ainda a necessidade de compreender melhor as relações sociais, pois estas envolvem diversas questões que vão além da equipe, paciente e família. Segundo Souza e Alves (2015) a abordagem multiprofissional é essencial tratando-se de plano de cuidados e, normalmente, é o enfermeiro que mais se aproxima do paciente e da família, por permanecer por mais tempo com estes, atuando 
como peça chave da equipe, sendo geralmente o profissional que consegue entrar no universo do paciente e de sua família.

Outro ponto destacado pelos profissionais é a necessidade da equipe em compreender que o cuidado deve ser oferecido de forma contínua ao paciente e aos familiares melhorando a qualidade de vida para ir além do apoio individualizado. No entanto, na prática, como o centro de atenção dos profissionais é voltada para o paciente que demanda cuidados dos especializados, as necessidades dos familiares acabam sendo às vezes pouco valorizada e/ou negligenciada. (MENEGUIM, RIBEIRO; 2016).

A pesquisa mostra que os familiares costumam ter sobrecarga de ordem física, emocional, espiritual, social e financeira, principalmente quando o tempo de cuidado é contínuo e a doença já se encontra em estágio avançado. Percebe-se também a falta de uma cultura de cuidado compartilhado entre os serviços de saúde distanciando do cuidado paliativo ideal, pois acaba dificultando a assistência que demandam os familiares. É muito importante que os profissionais entendam que o objetivo do cuidado no domicilio requer valorizar o conhecimento das diferentes pessoas envolvidas neste processo, que pode contribuir para melhor qualidade dessa assistência (MENIGUIM, RIBEIRO;2016)

A abordagem da equipe com os familiares é uma ação delicada que requer conhecimento técnico para a escuta, para um diálogo mais aberto e sincero, atendendo as necessidades e adquirindo confiança. O que é demonstrado pela fala de um participante:

"E abordagem acontece aos poucos, vamos conquistando a confiança e credibilidade para então chegarmos ao ponto certo. O cuidador aos poucos vai falando sobre seus medos, expectativas, necessidades, angústias, ...)” TE 1

Dentre os desafios relatados destacam-se aqueles advindos do próprio paciente ou da família, como por exemplo, a desistência de lutar pela vida, a demora de uma terapia medicamentosa causando maior sofrimento, o que são graves dificuldades para a equipe.

A maioria dos profissionais afirmou não utilizar nenhum instrumento de avaliação de cuidados paliativos, apenas dois profissionais referiram utilizar para 
avaliação algum método. Uma afirmou a utilização do plano de cuidados individualizado e outro faz o uso da escala de Paliative Performance Scale (PPS). Faz-se necessário conhecer e utilizar ferramentas capazes de organizar com mais qualidade o cuidado paliativo no domicilio dos pacientes e familiares. Além do PPS, que é um instrumento que permite obter indicações de sobrevida do paciente acompanhando a evolução da doença, pode ser utilizado também a Escala de Avaliação de Sintomas (EASAS) que é um questionário simples em que o paciente ou o cuidador marca em uma escala de 0 a 10 a presença de sintomas [nesta retição], e outra já bem conhecida pelos profissionais, a escala da dor. (VASCONCELOS,PEREIRA,2018). Estas ainda não estão claramente sistematizados na organização do processo destas equipes para o planejamento de ações em cuidados paliativos, o que reflete em uma exigência de perfil de atuação específica, com habilidades, conhecimentos e atitudes diferenciadas para a prática neste âmbito de cuidados. (SAVASSI, 2016).

Assim, a equipe procura manter uma comunicação efetiva e empática com os familiares quanto às expectativas propostas ao plano terapêutico do paciente:

“A equipe sempre se reúne com os familiares e esclarece o que são os cuidados paliativos explicando tudo que será feito e como se dará a indução ao paciente. A equipe sempre está à disposição dos familiares.” (E 1)

Entretanto, há múltiplos desafios para a qualidade de ações direcionadas à assistência domiciliar, como a carência de recursos para o cuidado paliativo, destacada como um dos pontos principais e, certificando assim a realidade em um sistema frágil, considerando a necessidade de envolvimento de toda a rede, para um apoio fundamental nas ações do cuidado em domicilio.

\section{Rede de cuidados: desafios e recomendações}

Conforme os relatos dos participantes da pesquisa os problemas enfrentados na atenção domiciliar para a prestação de cuidados são os mais diversos. A falta de recursos na rede assistencial é imensurável na visão de alguns profissionais, tanto em relação aos materiais de consumos e insumos, em equipamentos hospitalares, recursos humanos e nos medicamentos de maior consumo nos cuidados paliativos. Os discursos abaixo explicitam esta reflexão: 
“Ainda é precário, falta profissionais especializados, falta medicação, demora ao acesso às consultas, demora ao início de tratamento.” (TE 1).

"Uma das dificuldades é questão financeira, social e emocional. Falta de informação.” (AS 1).

“...nem sempre temos todo o material necessário para o atendimento. Exemplo: material de curativo, coberturas, pegamos muitos pacientes em situação de miséria." (AE 2).

Segundo Meneguin e Ribeiro (2016) há uma grande dificuldade em prestar a assistência da forma devida pelo não compartilhamento do cuidado conforme modalidades de atenção domiciliar do MS, pela falta de recursos humanos, de materiais, financeiro, na omissão dos serviços de saúde em referenciar os pacientes que são indicados para os cuidados paliativos na atenção domiciliar. Além disso, apontam a ESF despreparada e com déficit de profissionais para articular os cuidados demandados pela população no que tange os CP na atenção domiciliar, deixando evidente a falta de uma melhor estrutura da rede de atenção à saúde capaz de atender as necessidades das famílias.

Sobre o desafio referente aos recursos humanos para que a equipe de assistência domiciliar possa qualificar a prestação dos cuidados paliativos, encontra-se em várias literaturas o despreparo dos profissionais em lidar com a situação de morte (SILVA, 2018; CASTRO et al, 2018). Muito citado pelos participantes da pesquisa, foi a necessidade de capacitação dos profissionais, especialmente de nível técnico e a presença do profissional psicólogo na equipe. O psicólogo pode atuar no acompanhamento dos profissionais, do paciente e familiares e/ou cuidadores no momento que mais precisam ser acolhidos, ouvidos e amparados por um suporte emocional. (SOUZA E ALVES; 2015). O psicólogo busca a qualidade de vida, diminuindo o sofrimento, a ansiedade e depressão tanto do paciente quanto de seus familiares diante da terminalidade. A atuação do psicólogo tem grande importância tanto no nível de prevenção quanto nas variadas etapas do cuidar, contribuindo para que estes possam falar sobre a situação em que estão vivendo, com elaboração de um processo que os ajudará enfrentar a doença construindo experiências de adoecimento, processo de morte e luto. A escuta e o acolhimento são instrumentos indispensáveis para 
o psicólogo conhecer a real demanda do paciente para firmar uma relação de confiança (HERMES E LAMARCA, 2013; CERQUEIRA, 2016). Ainda Cerqueira (2016) aponta que a rede de atenção precisa ser reestruturada para melhoria na assistência psicológica, pois as demandas e a vagas ofertadas não condizem, deixando o paciente em espera ou direcionando-o para outro serviço da rede, não tendo uma contra-referência no retorno destes pacientes. Revela-se a necessidade de mais profissionais na assistência, melhores gestores à frente dos serviços e maior engajamento dos profissionais de psicologia.

Nos outros pontos da assistência que articulam com a atenção domiciliar há uma série de obstáculos que interferem no planejamento assistencial destes pacientes. $\mathrm{O}$ primeiro deles, em âmbito político, se refere à (des)organização da rede de cuidados, o que parece ter possibilidades de resolução, considerando o fato da não implementação da recente Política Nacional de Cuidado Paliativo para o SUS, aprovada em por meio da Resolução n.41, de 31 de outubro de 2018 (BRASIL, 2018; CASTRO, 2018; SOUZA 2017). Esta política vem ao encontro das necessidades para promover uma maior integração da RAS e uma assistência integral de CP em todos os pontos que compõe a assistência no âmbito do SUS.

Os participantes apontaram limites na relação com a atenção primária à saúde e a necessidade de leitos hospitalares de retaguarda para os cuidados paliativos, além da necessidade de hospital como espaço de cuidado qualificado em cuidados paliativos. Os trechos abaixo reforçam outras questões que dificultam o andamento do cuidado pelas equipes devido à fragmentação entre os pontos de assistência da RAS.

"Falta de recursos materiais, equipamentos, medicamentos." (AS 2).

“Consultas e exames de urgência para definição do diagnóstico, fornecimento de insumos, autorização p/ cirurgias de urgências." (E 2)

Souza e Alves (2015) corroboram com os achados da pesquisa ao apontarem os desafios que esta modalidade apresenta para praticar uma assistência integral, em decorrência da falta de organização de toda a rede que se faz de forma fragmentada faltando uma comunicação clara e objetiva que articula e programa os cuidados de forma a reduzir o tempo de espera e as possíveis pioras no quadro clinico do paciente. 
A articulação entre os diferentes pontos da rede de atenção são buscadas de diversas formas para os participantes da pesquisa: desde telefonemas, e-mails, relatórios, pelos profissionais das equipes da EMAD, e principalmente por intervenções da assistência social, de modo a tentar agilizar o processo e não prejudicar o andamento do cuidado. Mesmo assim, os participantes relatam a morosidade e precariedade da rede em um retorno positivo na maioria dos casos. Os profissionais deparam com situações socioeconômicas que interferem diretamente nos cuidados destes pacientes, uma vez que faltam recursos até para a própria alimentação familiar, ocasionando uma situação constrangedora e aumentando ainda mais os problemas afetivo-emocionais familiares. Geram ainda um desgaste emocional dos profissionais, interferindo na qualidade de vida dos que procuram prestar um cuidado de qualidade.

Outro aspecto bastante revelado como desafio se refere à abordagem familiar seja em decorrência da não adesão das famílias aos cuidados, sofrimento, carência socioeconômica, medo da morte no domicilio, dentre outros. Fica claro que a maior parte dos casos é a assistência social que articula o processo devido ao fator socioeconômico como o maior problema familiar, impactando nos cuidados paliativos domiciliares. Assim, os participantes afirmaram que a rede de atenção requer uma reestruturação para articular melhor a atenção domiciliar junto às unidades de atenção primária e a rede hospitalar, exigindo uma educação continuada, aprimorando cada vez mais os trabalhadores, melhorando as condições de trabalho. Nota-se a necessidade de políticas estruturais como as sociais e educacionais, investindo na melhoria da educação e qualidade de vida da população para que assim haja resultados positivos nos cuidados da atenção domiciliar. Esta reflexão corrobora com a necessidade de um sistema sanitário e social integrado, articulado com a comunidade e norteado por suas necessidades. Assim, sistemas integrados e o cuidado baseado na compaixão, na terapia da dignidade e da gentileza parecem apontar os caminhos para o cuidado paliativo em rede.

\section{Considerações finais}

Apesar da pouca adesão dos profissionais na pesquisa com possibilidade de ampliação dos achados que repercutem na implementação de políticas, na ampliação dos serviços e melhoria na prestação dos cuidados, ainda sim foi possível o cumprimento dos objetivos propostos. Merece destacar o aspecto benéfico na 
vinculação dos profissionais ao município uma vez que apenas um profissional não é efetivo, quesito positivo para uma assistência de qualidade por menor rotatividade dos profissionais, com maior vínculo com a população.

Ao final desse estudo foi possível confirmar que as equipes de $\mathrm{AD}$ em $\mathrm{CP}$, atuam de forma limitada por algumas questões que perpassam o fazer profissional. Além de expressarem a necessidade de capacitação voltada para o CP relataram também a necessidade de maiores investimentos na rede de assistência tanto em equipamentos, materiais de consumo e insumos e nas possibilidades junto das famílias, frente às questões sociais e econômicas vivenciadas por grande parte delas.

Foi percebida a necessidade de incremento ou inclusão da espiritualidade no ensino dos profissionais da saúde, ampliando sua visão no escopo do cuidado paliativo, aumentando a reciprocidade na relação profissional/paciente. A mudança na organização do processo de trabalho, e na conformação da equipe, uma vez que o profissional psicólogo não compõe todas as equipes da $\mathrm{AD}$ e que atuam nos $\mathrm{CP}$ cotidianamente, sendo reforçado pelos participantes a necessidade de um acompanhamento para si e os pacientes/familiares. Um maior envolvimento dos gestores na garantia dos recursos humanos e materiais que são fundamentais para o cuidado de qualidade foi revelado por todos os participantes.

\section{Referências}

ANTONECHEN Aline Cristina, DÓRO Maribel Pelaez. Qualidade de vida, ansiedade e depressão em pacientes de hemato-onco com dor crônica. Rev. Saúde Santa Maria, Jan/jun. 2016 v.42.nº 1p 225-234.

ANDRADE, Cristiani Garrido et al. Cuidados paliativos e comunicação: estudo com profissionais de saúde do serviço de atenção domiciliar. Revista de Pesquisa: Cuidado é Fundamental, jan/mar.2017(1):2015-< http://doi.org/109789/21755361.2017> Acesso em 20.mar. 2018.

ACADEMIA NACIONAL DE CUIDADOS PALIATIVOS. ANCP. Manual de Cuidados Paliativos. 2 Ed, 2012. 552 p.ARRIEIRA Isabel Cristina de Oliveira et al. Espiritualidade nos cuidados paliativos: experiência vivida de uma equipe 
interdisciplinar. Rev. Esc. Enf. USP, 2018, 52 e 03312 <. http:/dx.doi.org/101590/51980-220x.2017007403312>. Acesso em 25 nov.2018

BARBOSA, Silva Freitas, TROCHIN, Daisy Maria Rizatto. Manual de monitoramento da qualidade dos registros de enfermagem na assistência domiciliar. Rev. Bras. Enferm. mar/abr.2015;68(2):253-260.

BETIM. Prefeitura Municipal de Betim/Secretária Municipal de Saúde: Plano Municipal de Saúde de Betim. 2013. Disponível em < http://www.betim.mg.gov.br/ARQUIVOS_ANEXO/Plano_Municipal_de_Saude;;2015 0213.pdf>. Acesso em 22.mai.2018

BRASIL, Ministério da Saúde. Secretaria de Atenção à Saúde Departamento de Atenção Básica. (2012) Caderno de Atenção Domiciliar Volume 1 (Vol. Volume 1). 2013. Disponível em

http://189.28.128.100/dab/docs/portaldab/publicacoes/cad_vol2.pdf $>$. Acesso em 02 abr. 2018

BRASIL, Ministério da Saúde. Secretaria de Atenção à Saúde Departamento de Atenção Básica. (2013) Caderno de Atenção Domiciliar Volume 2. (Vol. Volume 2).2013, Disponível em:< http://189.28.128.100/dab/docs/portaldab/publicacoes/cad_vol2.pdf .> Acesso em 02 abr. 2019

BRASIL. Ministério da Saúde. Atenção domiciliar no SUS: resultados do laboratório de inovação em atenção domiciliar, 2014.

BRASIL, Ministério da saúde. Conselho nacional de saúde. Resolução no 41, de 31 de outubro de 2018. Dispõe sobre as diretrizes para a organização dos cuidados paliativos, a luz dos cuidados continuados integrados no âmbito SUS. Diário Oficial da União. Brasília n²09, 30 out. 2018, seção 1, p 57.

BRITO, Fabiana Medeiros et al. Cuidados paliativos e comunicação: estudo com profissionais de saúde do serviço de atenção domiciliar. Revista de Pesquisa: Cuidado é Fundamental, 2017. Online, 9(1), 215-221. https://doi.org/10.9789/21755361.2017.v9i1.215-221. Acesso em 04 abr. 2018 
CASTRO Edna Aparecida Barbosa, et al. Organização da atenção domiciliar com o Programa Melhor em Casa. Rev. Gaúcha Enferm. 2018;39:e2016-0002. <https://doi.org/10.1590/1983-1447.2018.2016-0002> Acesso em 10 nov.2018

Conselho Regional de Medicina de São Paulo CREMESP. Cuidado Paliativo. São Paulo 2008.689p

CERQUEIRA Maria Augusta Trindade. $\mathrm{O}$ atendimento psicológico na rede pública de saúde de salvador Brasil: os desafios da humanização, 2016, 90 f. Dissertação (Mestrado) Escola Baiana de Medicina e Saúde Publica

DALACORTE Roberta Rigo, et al Cuidados Paliativos em Geriatria e Gerontologia. São Paulo, Editora Atheneu2012

HERMES, Helida Ribeiro; LAMARCA, Isabel Cristina Arruda. Cuidados paliativos: uma abordagem a partir das categorias profissionais de saúde. Revista Ciência \& Saúde Coletiva. 2013, 18(9), 2577-2588.< https://doi.org/10.1590/S1413$81232013000900012>$. Acesso em 20 de mar. 2018

MACHADO, Jaqueline Holz et al.: Paciente que requer cuidados paliativos: percepção de enfermeiras. Enfermagem em foco, dez. 2015.6v, n1/4, p.67-71.

MATOS, Michele Rodrigues et al. Significado da atenção domiciliar e o momento vivido pelo paciente oncológico em cuidados paliativos. Rev. Eletr. Enf. [Internet], (Sentimentos relacionados a atenção domiciliar), 2016,18:e117910. Disponível em:< http://dx.doi.org/10.5216/ree.v18.35061>Acesso em: 20 mar 2018

MENEGUIM, Silmara; RIBEIRO, Rafaela. Dificuldades de cuidadores de pacientes em cuidados paliativos da estratégia em saúde da família. Texto contexto- Enferm, (Online) 2016; 25(1): 3360014. <http://dx.doi.org/10.1590/01040707201500003360014.> Acesso em 04 abr.2018.

MINAYO, Maria Cecilia Souza, DESLANDES, Suely Ferreira, GOMES, Romeu. Pesquisa Social: Teoria, Método e Criatividade. 25a ed.rev. Rio de Janeiro: Vozes, 2007. 
OLIVEIRA, Reinaldo Gomes. Blackbook- Enfermagem. Belo Horizonte. Ed. Blackbook, 2016

PESSINI, Leo; BERTACHINI, Luciana. Humanização e Cuidados paliativos. $5^{\circ}$ ed. São Paulo: Edições Loyola 2011.

PILATTI, Patrícia et al. Cuidados paliativos oncológicos em um serviço público de atenção domiciliar. Rev. Bras. Med. Fam. Comunidade. Rio de Janeiro, 12(39), 1-10. https://doi.org/10.5712/rbmfc12(39)1339. Acesso em 22 de mai. 2018

QUEIROZ, Ana Helena Araújo Bonfim et al. Percepção de familiares e profissionais de saúde sobre os cuidados no final da vida no âmbito da atenção primaria a saúde. Rev. Ciência \& Saúde Coletiva, Set 2013; 18(9):p2615.

RABELLO, Claudia Azevedo Ferreira Guimaraes; RODRIGUES, Paulo Henrique Almeida. Saúde da Família e cuidados paliativos infantis: Ouvindo os familiares de crianças dependentes de tecnologia. 2010, Ciência \& Saúde Coletiva, 15(2):31573166.

RIBEIRO, Aline Lima. et al. Enfermagem no cuidado paliativo domiciliar: o olhar do familiar do doente com câncer. Rev. Rene, 2014.

SAVASSI, Leonardo Cansado Monteiro. Os atuais desafios da Atenção Domiciliar na Atenção Primária à Saúde: uma análise na perspectiva do Sistema Único de Saúde. Revista Brasileira de Medicina de Família e Comunidade, 2016, Jan-Dez 11(38), 1-12.< https://doi.org/10.5712/rbmfc11(38)1259> . Acesso 08 mar, 2018

SILVA, Alexandre Ernesto. A produção de cuidados paliativos no contexto da atenção domiciliar. 2018169 f. Tese (doutorado): Universidade Federal de Minas Gerais, Escola de Enfermagem, Belo Horizonte.

SILVA, Mariana Lobato Santos Silva. O papel do profissional de Atenção Primaria à Saúde em cuidados Paliativos. Rev. Bras. Med. Farm. Comunidade. Rio de Janeiro, $2014 ; 9(30): 45-53.15$. 
SOUZA, Janaina Meirelles; ALVES, Elioenai Dornelles. Cuidados paliativos de Enfermagem da atenção domiciliar. Rev. Enferm. UFPE. Recife, 2015, 9(2): 669676.

SOUZA, Janaina Meirelles; ALVES, Elioenai Dornelles. Competências do enfermeiro para o Cuidado Paliativo na Atenção Domiciliar. Acta Paul Enf. 2015; 28(3):264269. <http://dx.doi.org/10.1590/1982-0194201500044.> Acesso em: 05 mai. 2018

VASCONCELOS, Gabriela Belém; PEREIRA, Patrícia Maria. Cuidados paliativos em atenção domiciliar: uma revisão bibliográfica. Rev. Adm. Saúde,2018 18(70).

VILAS BOAS, Maria Leopoldina Castro; SHIMIZU, Helena Eri; SANCHES, Mauro Niskir. Elaboração de instrumento de classificação de complexidade assistencial de pacientes em atenção domiciliar. Rev. Esc. Enf. USP.2016;50(3):433-439. <http://dx.doi.org/10.1590/S0080-623420160000400009.>Acesso em: 20 Mai. 2018.

WHO. World Health Organization. Definition of Palliative care. Disponível em: <http://www.who.int/cancer/palliative/definition/en> Acesso em: 26 Jun. 2018. 